\title{
Subpicotesla scalar atomic magnetometer with a microfabricated cell
}

Cite as: J. Appl. Phys. 126, 124503 (2019); doi: $10.1063 / 1.5113520$

Submitted: 5 June 2019 . Accepted: 4 September 2019 .

Published Online: 26 September 2019

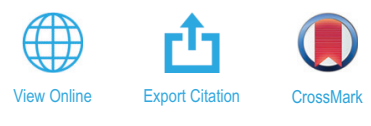

Rui Zhang, ${ }^{1, \text { a) }}$ (D) Terry Dyer, ${ }^{2, b)}$ Nathan Brockie, ${ }^{2, c)}$ Roozbeh Parsa, ${ }^{3, d)}$ and Rahul Mhaskar ${ }^{1}$

\author{
AFFILIATIONS \\ ${ }^{1}$ Ceometrics Inc., San Jose, California 95131, USA \\ ${ }^{2}$ Texas Instruments, Greenock PA16 OEQ, United Kingdom \\ ${ }^{3}$ Texas Instruments, Santa Clara, California 95051, USA \\ a)Email: rzhang@geometrics.com

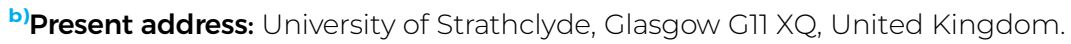 \\ ${ }^{c)}$ Present address: Nu Nano Ltd, Bristol BS2 OXJ, United Kingdom. \\ d) Present address: Rockley Photonics Inc., San Jose, California 95110, USA.
}

\begin{abstract}
We demonstrated a scalar atomic magnetometer using a microfabricated Cs vapor cell. The atomic spin precession is driven by an amplitude-modulated circularly-polarized pump laser resonant on the D1 transition of Cs atoms and detected by an off-resonant linearlypolarized probe laser using a balanced polarimeter setup. Under a magnetic field with amplitude in the Earth's magnetic field range, the magnetometer in the gradiometer mode can reach sensitivities below $150 \mathrm{fT} / \sqrt{\mathrm{Hz}}$, which shows that the magnetometer by itself can achieve sub-100 fT $/ \sqrt{\mathrm{Hz}}$ sensitivities. In addition to its high sensitivity, the magnetometer has a bandwidth close to $1 \mathrm{kHz}$ due to the broad magnetic resonance inside the small vapor cell. Our experiment suggests the feasibility of a portable low-power and high-performance magnetometer which can be operated in the Earth's magnetic field. Such a device will greatly reduce the restrictions on the operating environment and expand the range of applications for atomic magnetometers, such as detection of nuclear magnetic resonance in low magnetic fields.
\end{abstract}

Published under license by AIP Publishing. https://doi.org/10.1063/1.5113520

\section{INTRODUCTION}

Atomic sensors have been well known for their ultraprecise measurement of many important physical quantities, ${ }^{1}$ such as time, angular velocity, and magnetic field. However, they have not been widely used yet in commercial applications mostly due to their high cost, large size, and high power consumption. In recent years, much progress has been made toward miniaturization of atomic sensors, ${ }^{2}$ especially in atomic clocks. ${ }^{3}$ The first chip scale atomic clock became commercially available in 2011. Chip scale atomic magnetometers, constructed using the technique of Micro-ElectroMechanical Systems (MEMS), have also been demonstrated in laboratories. ${ }^{4}$ MEMS scalar atomic magnetometers can reach sensitivities of $5 \mathrm{pT} / \sqrt{\mathrm{Hz}}$. More recently, using a MEMS-fabricated vapor cell, researchers achieved sensitivity below $100 \mathrm{ft} / \sqrt{\mathrm{Hz}}$ with an atomic magnetometer operating in the spin-exchange-relaxation-free (SERF) regime. ${ }^{5}$ Advances in MEMS based research can eventually lead to small, low-cost, and power efficient atomic magnetometers since the
MEMS technology not only produces much smaller devices but also makes mass production possible at reduced costs. Here, we demonstrate a scalar atomic magnetometer using a MEMS-based Cs vapor cell. The achieved sensitivity is comparable to the SERF MEMS magnetometer with the advantage of being operational in a much larger and wider range of magnetic fields. Similar results were recently demonstrated in a slightly smaller $\mathrm{Rb}$ vapor cell with about $300 \mathrm{ft} / \sqrt{\mathrm{Hz}}$ sensitivity ${ }^{6}$ and using much bigger cells scalar magnetometers with better than $20 \mathrm{ft} / \sqrt{\mathrm{Hz}}$ sensitivity have been achieved. $^{7}$ Comparable sensitivity performance is also expected for a recently proposed atomic magnetometer system. ${ }^{8}$

\section{EXPERIMENTAL SETUP}

Our experimental setup is shown schematically in Fig. 1. Pump and probe beams are originated from two distributed Bragg reflector (DFB) lasers. The pump light is circularly polarized and passed through an acousto-optical modulator (AOM) before 


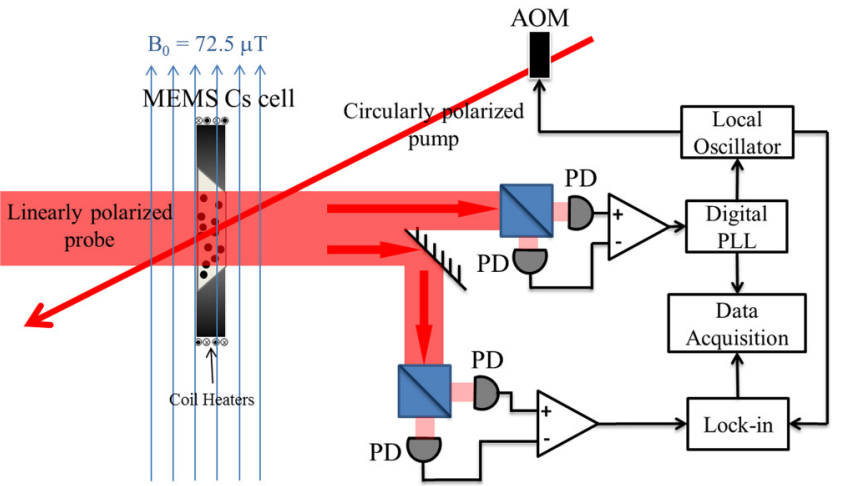

FIG. 1. Schematics of the experimental setup for the gradiometer measurement using a MEMS cell. The cell has an inner dimension of $4.5 \times 3 \times 1.5 \mathrm{~mm}^{3}$. The probe and pump beams are counterpropagating and cover the $4.5 \times 3 \mathrm{~mm}^{2}$ window of the cell. After the cell, the probe beam is split into two parts for the gradiometer measurement.

overlapped with the linearly polarized probe beam at the Cs vapor cell, which is located inside a 4-layer magnetic shield can (not shown). The Cs vapor cell was manufactured by Texas Instruments using the MEMS technology. The cell has a trapezoidal structure with minimum inner dimensions $4.5 \times 3 \times 1.5 \mathrm{~mm}^{3}$, the shortest distance being in the light path direction. The cell is also filled with about 50 Torr nitrogen gas as the standard buffer gas to reduce the collision between the Cs atoms and the walls. The cell is heated to about $110^{\circ} \mathrm{C}$ by running an $\mathrm{AC}$ current $(10 \mathrm{kHz})$ through external coils. The coils are wound such that the AC current generates minimal stray magnetic field. The pump and probe beams are counterpropagating and have a Gaussian beam waist of $3 \mathrm{~mm}$ and $4 \mathrm{~mm}$ in diameter, respectively (in Fig. 1, beam sizes are not shown proportionally). The pump light has $1 \mathrm{~mW}$ in the total power before the cell with AOM continuously on $(0.6 \mathrm{~mW}$ after the cell at room temperature) and its frequency is tuned to the $|F=3\rangle>\rightarrow \mid F^{\prime}=4>$ transition of the Cs D1 line. When the pump light is on, a spin polarization in the $\mid \mathrm{F}=4>$ state is generated. In the presence of a magnetic field perpendicular to the light, the atomic ground state spin undergoes the Larmor precession. When the frequency of the periodic pumping, controlled by the amplitude modulation (AM) of the AOM, is synchronized with the Larmor precession frequency, the spin population has a resonance, called the magnetic resonance. The magnetic resonance can be detected by the probe light. The probe light is linearly polarized, $0.7 \mathrm{~mW}$ in the total power before the cell and about $4 \mathrm{GHz}$ blue detuned with respect to the $|\mathrm{F}=4>\rightarrow| \mathrm{F}^{\prime}=3>$ transition of the Cs D1 line. After passing through the Cs cell, the probe light power is reduced to about $210 \mu \mathrm{W}$ and is spatially split into two beams, each entering a standard balanced polarimeter setup for the detection of the Larmor precession. The differential photodiode currents are then amplified through transimpedance amplifiers with a gain of $100 \mathrm{k} \Omega$. Out of the amplifier, one of the Larmor precession signals is fed into a lock-in amplifier and the other into a digital phase lock loop (PLL). When the PLL is enabled and its local oscillator signal is fed into the reference input of the lock-in amplifier, the output of the lock-in amplifier becomes the differential reading between the two Larmor precession signals, thus the gradiometer measurement. A similar setup was previously discussed.

\section{EXPERIMENTAL RESULTS}

When the PLL is not enabled, the pump AM frequency can be scanned around the Larmor frequency and the outputs of the lock-in amplifier can be recorded as a function of the pump AM frequency. In the presence of a perpendicular magnetic field of $\mathrm{B}_{0}=72.5 \mu \mathrm{T}$, generated by a pair of Helmholtz coils inside the shield can, the observed magnetic resonance is shown in Fig. 2(a). The phase of the lock-in amplifier is adjusted such that the quadrature component is zero (has the maximum slope) at the resonance. The resonance has a FWHM of about $1.8 \mathrm{kHz}$, and the slope of the quadrature component is about $1.35 \mathrm{mV} / \mathrm{Hz}$ at the resonance.
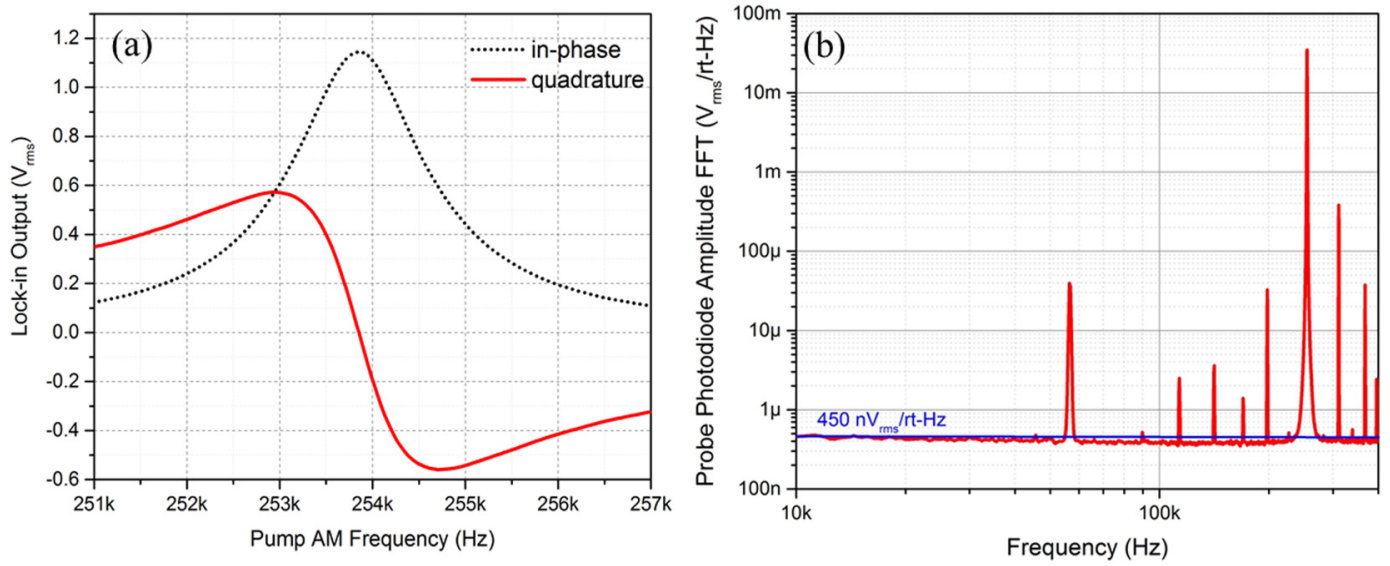

FIG. 2. (a) Probe lock-in signal (r.m.s. amplitude) as a function of pump AM frequency f. The black dotted (red solid) curve represents the in-phase (quadrature) component. (b) Amplitude spectral density of the probe photodiode signal after the transimpedance amplifier. 
To measure the magnetic field in the open loop mode, the pump $\mathrm{AM}$ frequency is set at the center frequency, $f_{0}$, of the magnetic resonance. Then, the quadrature output of the lock-in amplifier can be converted to the magnetic field $\mathrm{B}$ according to the following equation: $\mathrm{B}=\left(f_{0}+\mathrm{V} / \mathrm{L}\right) / \mathrm{k}$, where $\mathrm{V}$ is the quadrature output, $\mathrm{L}=1.35 \mathrm{mV} / \mathrm{Hz}$ is the slope, and $\mathrm{k}=3.5 \mathrm{~Hz} / \mathrm{nT}$ is the Zeeman splitting factor between two adjacent magnetic sublevels of Cs atoms in the $\mid \mathrm{F}=4>$ ground state. Similarly, the magnetometer output noise can be measured in the open mode as $\delta \mathrm{B}=(\delta \mathrm{V} / \mathrm{L}) / \mathrm{k}$, where $\delta \mathrm{V}$ is the quadrature output noise. The amplitude spectral density of the amplified probe photodiode signal is plotted in Fig. 2(b), the noise floor of which is a good estimation of $\delta \mathrm{V}$. Based on the $450 \mathrm{nV} / \sqrt{\mathrm{Hz}}$ noise floor shown in Fig. 2(b), the magnetometer output noise should be around $95 \mathrm{ft} / \sqrt{\mathrm{Hz}}$. However, here we assume that $f_{0}$ is constant, which is often not the case, especially for a magnetic field as strong as the one we are using. The current noise of the electronics that generates $B_{0}$ is often the dominating noise. Therefore, to measure the fundamental noise of the magnetometer, we need to use the gradiometer setup shown in Fig. 1.

To operate the system in the gradiometer mode, the PLL is enabled to track the magnetic field $B_{0}$. The PLL output, $f_{\mathrm{PLL}}=f_{0}+\Delta f$, contains both the fluctuation of the $\mathrm{B}_{0}$ and a slight frequency offset due to the magnetic field gradient. When $f_{\mathrm{PLL}}$ is fed into the reference input of the lock-in amplifier, the common fluctuation in $f_{0}$ is canceled, leaving behind only the fundamental noise of the magnetic field gradient. The fundamental noise of the gradient is a factor of $\sqrt{2}$ larger than that of an individual magnetometer, assuming the same fundamental noise for both paths. In the gradiometer experiment, we record both the PLL output (individual magnetometer reading) and the lock-in amplifier output (magnetic field gradient) and compare their noise levels, shown in Fig. 3 as the black curve and the cyan curve (overlapping with the red curve at low frequencies), respectively. As we can see, the gradiometer output has a noise level as low as $150 \mathrm{ft} / \sqrt{\mathrm{Hz}}$, much lower than the individual magnetometer measurement. The fundamental noise of each individual magnetometer should be around $105 \mathrm{ft} / \sqrt{\mathrm{Hz}}$ based on the gradiometer result, which is very close to the $95 \mathrm{ft} / \sqrt{\mathrm{Hz}}$ estimation based on the noise floor of Fig. 2(b). Photon shot noise is often the most dominant noise source of the atomic magnetometer. For a light power of $p$, the photon shot noise has a flat amplitude spectral density of $\sqrt{(2 h v p)}$, where $h v$ is the single photon energy. With $p=105 \mu \mathrm{W}$, $\lambda=895 \mathrm{~nm}$, photodiode efficiency of $0.65 \mathrm{~A} / \mathrm{W}$, and $100 \mathrm{k} \Omega$ transimpedance amplifier gain, the noise floor of the amplitude spectral density due to the photon shot noise is $446 \mathrm{nV} / \sqrt{\mathrm{Hz}}$, leading to a magnetometer noise of about $94 \mathrm{ft} / \sqrt{\mathrm{Hz}}$. Therefore, the fundamental scalar magnetometer noise is indeed dominated by the photon shot noise.

The probe beam is split into two for the gradiometer measurement. If the full probe beam is used, there should be another factor of $\sqrt{2}$ improvement in the fundamental scalar magnetometer noise, provided that the noise of the individual magnetometer shown in Fig. 3 is dominated by fluctuations in the background magnetic field. Based on the noise floor shown in Fig. 2(b), the individual scalar magnetometer noise is not due to the noise in the probe or pump beams, leaving the background magnetic field

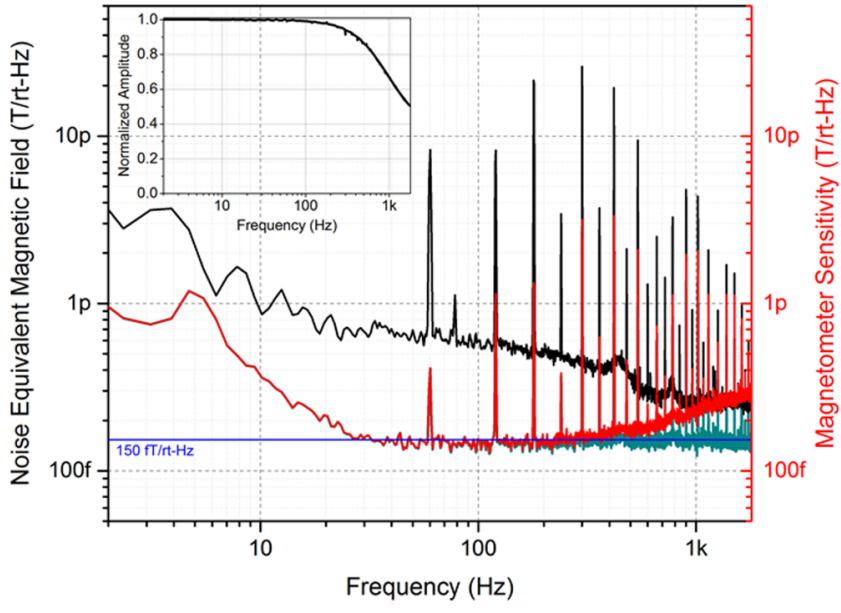

FIG. 3. Spectral density of the magnetometer noise in the gradiometer mode (cyan curve, overlapping with the red curve at low frequencies). The inset shows the bandwidth of the magnetometer in the open loop mode. The red curve is the fundamental sensitivity of the magnetometer, which represents the minimum signal level detectable by the magnetometer with a signal to noise ratio of 1:1. The black curve is the noise spectral density of the individual magnetometer operating in the phase-lock-loop mode.

fluctuation the most likely cause. With a better current source for the background magnetic field, we are able to achieve better than $180 \mathrm{ft} / \sqrt{\mathrm{Hz}}$ noise level for the individual scalar magnetometer. Therefore, we are confident that we should be able to achieve better than $100 \mathrm{ft} / \sqrt{\mathrm{Hz}}$ noise performance with the microfabricated cell. The increase in the noise level below $30 \mathrm{~Hz}$ shown in Fig. 3 is mainly due to air current along the beam paths, causing fluctuations of the air refractive index, which affects the polarization of the beams. This negative effect can potentially be eliminated using the vacuum packaging technique. The sharp spikes in Fig. 3 are due to the couplings of the $60 \mathrm{~Hz}$ power line radiation and its harmonics into the electronics.

Another important performance characteristic of a magnetometer is its bandwidth. To measure the bandwidth, we have another set of Helmholtz coil in the same direction as $B_{0}$, which can independently generate an oscillating magnetic field, $B_{1}$ sin $\left(2 \pi f_{1} t\right)$. We fix the amplitude $B_{1}$ at about $3 n T$ and vary the frequency $f_{1}$. The oscillating magnetic field can be detected by the magnetometer in the open mode and the measured amplitude $B_{1}$ as a function of $f_{1}$ is recorded. The normalized $B_{1}$ vs $f_{1}$ is plotted in Fig. 3 inset. As shown, the $3 \mathrm{~dB}$-point of the bandwidth curve is about $900 \mathrm{~Hz}$. The bandwidth is limited by the width of the magnetic resonance shown in Fig. 2. By combining the magnetometer noise spectrum and its bandwidth, we can achieve the sensitivity of the magnetometer. We define the sensitivity as the minimum detectable signal level by the magnetometer at a signal to noise ratio of 1:1. According to this definition, the sensitivity curve can be calculated by dividing the noise spectrum by the bandwidth curve, which is shown as the red curve in Fig. 3. At low frequencies, the sensitivity and the noise curves overlap. At higher frequencies where the signal 
starts to drop due to the bandwidth, the sensitivity is getting worse since the noise remains almost the same. Based on the sensitivity curve, for a $300 \mathrm{ft} / \sqrt{\mathrm{Hz}}$ performance, the gradiometer can be operated up to $2 \mathrm{kHz}$.

\section{DISCUSSION AND CONCLUSION}

The combination of high sensitivity, large bandwidth, and operation in the Earth's magnetic field, in addition to the possibility of small size and low power consumption associated with the microfabricated cell, makes the demonstrated magnetometer attractive to many applications. ${ }^{10}$ For example, using the atomic magnetometer for the detection of nuclear magnetic resonance (NMR) in low magnetic fields ${ }^{11}$ is becoming an interesting research topic due to many advantages brought forth by the low operational magnetic field and the compact size of the atomic magnetometers. However, one of the major challenges arises due to the several orders of difference between the gyromagnetic ratios of electrons and nucleus. ${ }^{12}$ The NMR signal often falls far outside the bandwidth of the atomic magnetometer if the NMR sample and the magnetometer are in the same magnetic field. People have used smart ways to circumvent this difficulty, ${ }^{11}$ but at the cost of the increased complexity of the setup. A highly sensitive scalar magnetometer with near $1 \mathrm{kHz}$ bandwidth and using a microfabricated cell can potentially solve many problems in the NMR application. First, due to the high bandwidth, the magnetometer and the NMR nucleus can be operated in the same magnetic field. Taking one of the commonly used nuclei in NMR, ${ }^{129} \mathrm{Xe}$ for example, at a magnetic field of $50 \mu \mathrm{T}$, the nucleus precession has a frequency of about $600 \mathrm{~Hz}$, well within the bandwidth of the scalar magnetometer. Second, the nucleus can be brought much closer to the magnetometer since now they can be in the same magnetic field. Combined with the microfabricated cell and the microfluid channels, ${ }^{13}$ it is possible to reduce the separation between the cell and nucleus to within $1 \mathrm{~mm}$, greatly enhancing the NMR signal. In addition, the scalar magnetometer can be operated in a wide range of magnetic fields, offering tuning of the NMR frequency if necessary. Depending on the strength of the NMR signal, NMR detection may become possible in an unshielded environment using the magnetometer in the gradiometer mode. The demonstrated scalar atomic magnetometer may well lead to a portable and low-cost NMR device in the near future.

\section{REFERENCES}

${ }^{1}$ J. Kitching, S. Knappe, and E. A. Donley, IEEE Sens. J. 11, 1749 (2011).

2J. Kitching, Appl. Phys. Rev. 5, 031302 (2018).

${ }^{3}$ S. Knappe, V. Shah, P. D. D. Schwindt, L. Hollberg, J. Kitching, L.-A. Liew, and J. Moreland, Appl. Phys. Lett. 85, 1460 (2004); S. Knappe, P. Schwindt, V. Shah, L. Hollberg, J. Kitching, L. Liew, and J. Moreland, Opt. Express 13, 1249 (2005); S. Knappe, P. D. D. Schwindt, V. Gerginov, V. Shah, L. Liew, J. Moreland, H. G. Robinson, L. Hollberg, and J. Kitching, J. Opt. A Pure Appl. Opt. 8, S318 (2006).

${ }^{4}$ P. Schwindt, S. Knappe, V. Shah, L. Hollberg, J. Kitching, L. A. Liew, and J. Moreland, Appl. Phys. Lett. 85, 6409 (2004); P. Schwindt, B. Lindseth, S. Knappe, V. Shah, J. Kitching, and L. A. Liew, Appl. Phys. Lett. 90, 081102 (2007).

${ }^{\mathbf{5}}$ V. Shah, S. Knappe, P. D. D. Schwindt, and J. Kitching, Nat. Photonics 1, 649 (2007).

${ }^{\mathbf{6}}$ V. Gerginov, S. Krzyzewski, and S. Knappe, J. Opt. Soc. Am. B 34, 1429 (2017).

${ }^{7}$ S. J. Smullin, I. M. Savukov, G. Vasilakis, R. K. Ghosh, and M. V. Romalis, Phys. Rev. A 80, 033420 (2009); D. Sheng, S. Li, N. Dural, and M. V. Romalis, Phys. Rev. Lett. 110, 160802 (2013); V. G. Lucivero, W. Lee, M. E. Limes, E. L. Foley, T. W. Kornack, and M. V. Romalis, Quantum Information and Measurement, p. T3C.3 (2019).

${ }^{8}$ M. D. Bulatowicz, T. G. Walker, M. S. Larsen, U.S. Patent US2,018,037,281,3A1 (27 December 2018).

${ }^{9}$ G. Bevilacqua, V. Biancalana, P. Chessa, and Y. Dancheva, Appl. Phys. B 122, 103 (2016).

${ }^{10}$ D. Budker and M. Romalis, Nat. Phys. 3, 227 (2007).

${ }^{11}$ V. V. Yashchuk, J. Granwehr, D. F. Kimball, S. M. Rochester, A. H. Trabesinger, J. T. Urban, D. Budker, and A. Pines, Phys. Rev. Lett. 93, 160801 (2004); I. M. Savukov and M. V. Romalis, Phys. Rev. Lett. 94, 123001 (2005); I. M. Savukov, S. J. Seltzer, and M. V. Romalis, J. Magn. Reson. 185, 214 (2007); M. P. Ledbetter, I. M. Savukov, D. Budker, V. Shah, S. Knappe, J. Kitching, D. J. Michalak, S. Xu, and A. Pines, Proc. Natl. Acad. Sci. U.S.A. 105, 2286 (2008); M. P. Ledbetter, C. W. Crawford, A. Pines, D. E. Wemmer, S. Knappe, J. Kitching, and D. Budker, J. Magn. Reson. 199, 25 (2009); G. Bevilacqua, V. Biancalana, Y. Dancheva, and L. Moi, J. Magn. Reson. 201, 222 (2009); T. Theis, P. Ganssle, G. Kervern, S. Knappe, J. Kitching, M. P. Ledbetter, D. Budker, and A. Pines, Nat. Phys. 7, 571 (2011); M. P. Ledbetter, T. Theis, J. W. Blanchard, H. Ring, P. Ganssle, S. Appelt, B. Blümich, A. Pines, and D. Budker, Phys. Rev. Lett. 107, 107601 (2011); G. Bevilacqua, V. Biancalana, A. B.-A. Baranga, Y. Dancheva, and C. Rossi, J. Magn. Reson. 263, 65 (2016).

${ }^{12}$ D. Budker and D. F. J. Kimball, Optical Magnetometry (Cambridge University Press, 2013), p. 268.

${ }^{13}$ R. Jimenez-Martınez, D. J. Kennedy, M. Rosenbluh, E. A. Donley, S. Knappe, S. J. Seltzer, H. L. Ring, V. S. Bajaj, and J. Kitching, Nat. Commun. 5, 3908 (2014). 